\title{
Function Knowledge Retrieval Based on Functions Knowledge Ontology
}

\author{
Baoru Su ${ }^{1, a}$, Yong Liu ${ }^{1, b}$ \\ ${ }^{1}$ The College of Information Science and Technology, \\ Qingdao University of Science \& Technology, Qingdao \\ 266061, Shandong, China \\ asubaoru65@aliyun.com, ${ }^{b}$ liuyong020202@163.com
}

\begin{abstract}
It has been found that design knowledge management is facing many problems in product design. In order to improve the design of knowledge sharing and reusability, the research of function knowledge ontology model has been carried out, which include extended device ontology and way of function achievement. Based on the combination above a refrigerator function ontology and function decomposition tree has been set up. Function knowledge retrieval on constructing the function ontology has been implemented. A software platform for sharing knowledge function decomposition tree has been designed.
\end{abstract}

Keywords- Function knowledge ontology; Manufacturing; Knowledge representation; Function decomposition tree

\section{INTRODUCTION}

Product design is a key stage to determine product success. However, design knowledge management is facing many problems now. The current situation of design knowledge expression is as following:

- The designer access difficulties.

- Searching for many documents, the manuals and data occurred in the development process is time-consuming, and the text data about description of concept is disunity, no semantic expression, it is difficult for computer to understand.

- Capture of implicit knowledge is not easy.

Main reason for the above problems lies in the understanding insufficiency of the function of generating aspect, thus leading to the different views about object world in different application fields and the lack of consistency in the function of knowledge account. In order to achieve the knowledge sharing and reuse in different field, the function knowledge ontology framework is used in design.

In the design activities, the design knowledge mainly including:

- Function knowledge:

It is used to describe the tasks of products, describe the functions and sub functions of product. Function description includes content of function, the implementation parameters of function, performance index of function and so on.

- Technical theory knowledge.

Describe the principle of product functions and sub functions. Its expression is more complicated, on one hand; its description can be expressed in text, data and parameters explanation, on the other hand, to have graphics support product principle solution.

- Structure knowledge:

It is used to describe the structure design of products, it is the refinement and expansion of principle, it is the structure carrier of theory of solving domain, and it is used to describe the shape, size and parameters of the key parts of the product.

Mapping of product function and structure is solving structure to achieve the function model of product; the functional description of product will be changed to the description of how to realize these functions with the specific switch, size and the relationship between the parts of components.

The objective of structure realization is Abstraction of the product structure; and the structure is a set of components or elements to realize some functions. The mapping relationship between function and structure is many-to-many. A function may be implemented by one or more features or components, and a feature or element may also be performing one or more functions.

- Examples knowledge.

Failure examples of the design, including the scheme design cases, the product structure knowledge examples, technical theory examples and so on. It contains more actual factors, and it is foundation of the analog design and case based reasoning design.

Function ontology theory is applied to manufacturing industry in this paper, function knowledge modeling method is studied, theory system and construction method of function concept ontology are introduced, and the principle of functional decomposition tree is introduced as well.

This paper draws the function ontology theory presented by Mizoguchi lab of the Osaka University in Japan, Ontology is introduced in the home appliance manufacturing industry (refrigerator as an example), so that an accurate, efficient, and intelligent retrieval of function knowledge is implemented.

\section{FUNCTION ONTOLOGY}

Functional ontology is proposed based on studying about ontology, ontology in the application of knowledge engineering and F-B-S architecture. At present, no one does further research in this field in China, only a few scholars who have done some research of computer integrated manufacturing technology tried to use ontology to solve some product knowledge representation and information modeling, representatives are as follows:

Dr. Li Jian of Beijing University of Aeronautics and Astronautics [1] put forward the concept of principle 
component and functional connectivity through the analysis of the function of the product element, working principle and components of functional surfaces, and set up product principle structure model on this basis using object oriented method, product design process model based on function are given as well.

Dr. Shi Dongyan of Harbin Institute of Technology [2], put forward the concept of design process based on function through the analysis of the research and application of conceptual design process theory, studies the application of ontology knowledge in conceptual design process by referencing ontology. Through the analysis of existing problems in function definition, proposed the function can be expressed using the function of semantic; proposed uses the ontology theory to establish the conceptual design solving process of domain knowledge. Associate professor Wang Youyuan of the Nanjing University of Aeronautics \& Astronautics [3], realize the share and reuse the design knowledge through the research of conceptual design product information modeling. Analysis of the F-B-S framework of information of product conceptual design representation, proposes the conceptual design product information modeling method based on ontology.

Above scholars' Research only remain in the application field; no one has done some research in theory field in China. In abroad, the representative research institution about function of Ontology is Mizoguchi Research Laboratory of Osaka University in Japan. They made a deep study of the ontology and F-B-S schema, and successfully applied in the manufacturing field, presents a hierarchical framework of ontology, knowledge and the function model [4][5] as Figure 1, they studied the function and the function concept around the center of equipment and put forward FTs and FBRL[6] in order to realize the function behavior mapping. Their research has been successfully applied to engineering, chemical plants, oil refineries, power plants, washing machine and transistor factory and other fields, design and implementation of knowledge sharing and reuse are achieved.

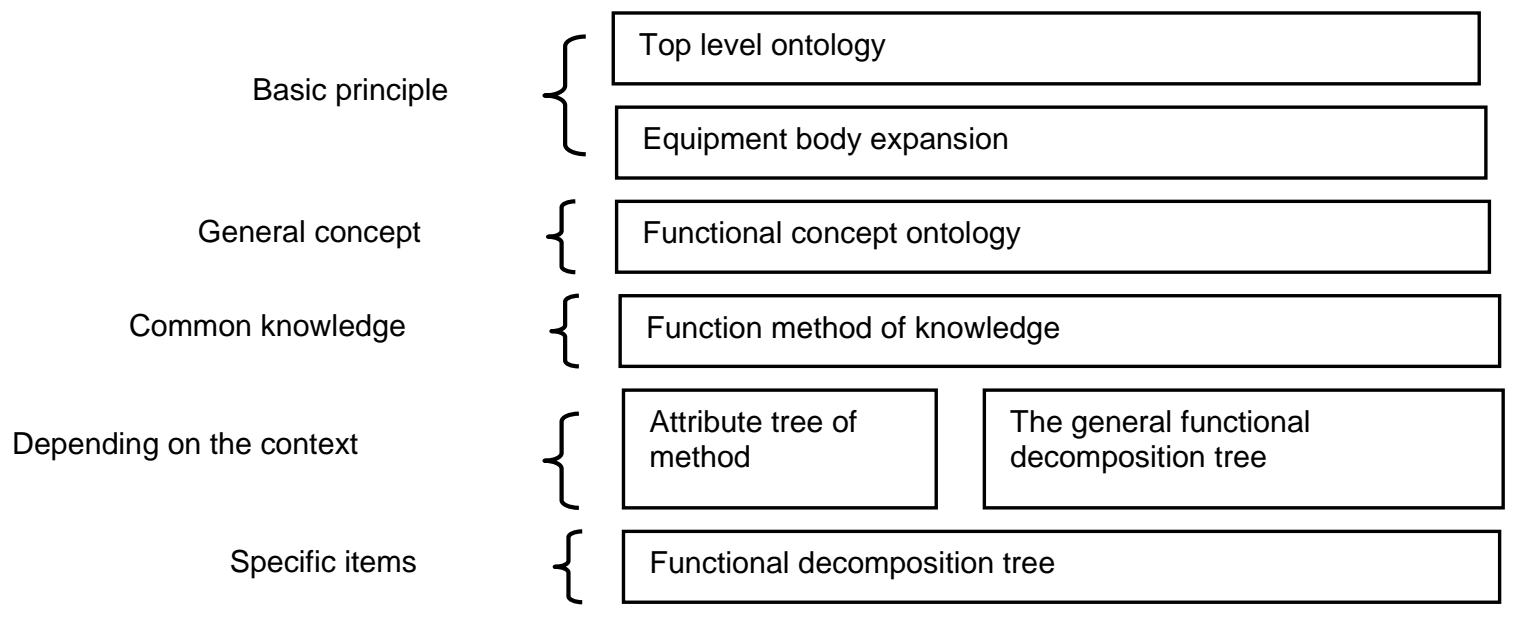

Figure 1. Hierarchy framework of Function, Knowledge and Ontology

To sum up, the function ontology is based on the ontology principle, achieving Standardization of function knowledge in conceptual design field of products, so as to effectively share the function knowledge, and realize the function knowledge reusability. The function ontology defines the function space in the conceptual vocabulary to specifications and limited function space. Function concept of application in ontology enables designers to better understand the design demand and effective reasoning in function space.

\section{APPLICATION OF FUNCTION ONTOLOGY IN THE FIELD OF HOME APPLIANCES}

\section{A Design}

Through the research and analysis on the function knowledge framework, we found that functional decomposition tree contains the design concept and design principle of equipment; and because the function concept came from functional concept ontology, so the problem of inconsistent terminology is solved. If the engineer can express design principle of equipment using functional decomposition tree after the completion of the design, then it is easy to realize the sharing of design knowledge. In this paper, the design and implementation of ontology based retrieval function are achieved, Firstly create the ontology library, and then the Jena inference engine reasoning according to reasoning rules designed, system is expected to achieve a relevant function of the retrieval platform. Users only need to input the query component function, and get a component with the function, users also can search all the functions of a member, user can also retrieve a method of function realization, agent and the entity function through this platform. The system integrated three body mentioned 
in reference [4][5] into one body, which is conducive to the ontology query.

\section{$B$ The construction of function ontology}

In a previous study, the ontology frame is built by Hozo software. Hozo is an ontology editing tools developed by Mizoguchi lab of Osaka University, Japan, a visual frame oriented structure, the user can get good visual mode through the construction of Hozo ontology immediately and there is a clear display of the relationship between the concepts. However, because it does not support multiple inheritance and description of example is not very detailed, so the system built ontology using protégé. to describe and express the field of home appliances (taking refrigerators for example) related factors function Objectively and accurately has important application significance to knowledge extraction, knowledge retrieval, post design etc..

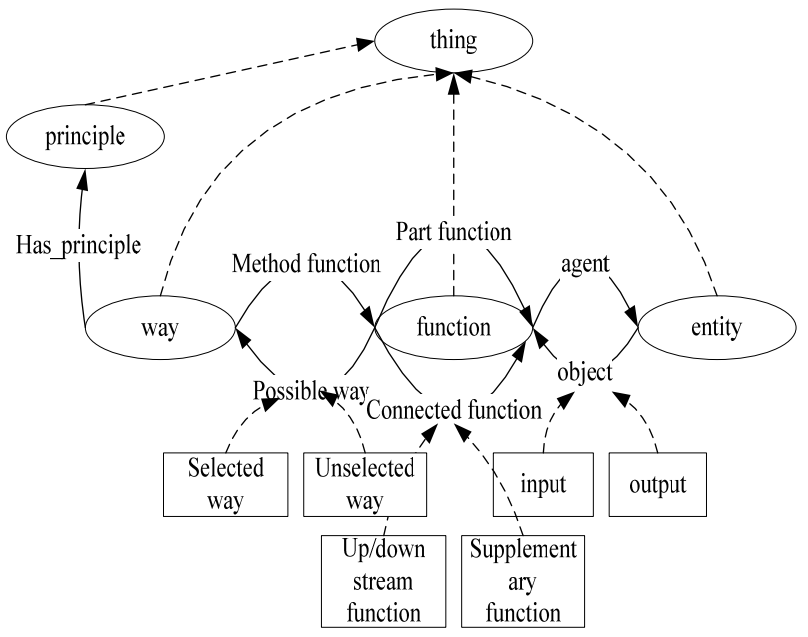

Figure 2. Function Ontology Framework
In this paper, the scientific research as a target, with concise, avoid unnecessary redundancy and convenient for extracting the operable principle, borrowing the function ontology framework proposed by Mizoguchi lab, according to the application needs of home appliances field, the object and relationship in appliance manufacturing process are analyzed objectively, Combining with the related information, an important object of appliance design process and relationship is refined and the refrigerator ontology for the object of study is constructed as Figure 2.

The main concept includes for categories: function, implementation method, the implementation principle and entity. More specific concept can be further subdivided. In order to extract and manage the relationship between objects, reflect structure and its rule in the design process, the attributes and relationships of objects is also described in constructing refrigerator ontology. as Figure 3. 4, an .Owl file is output after ontology construction as so to program query on the document.

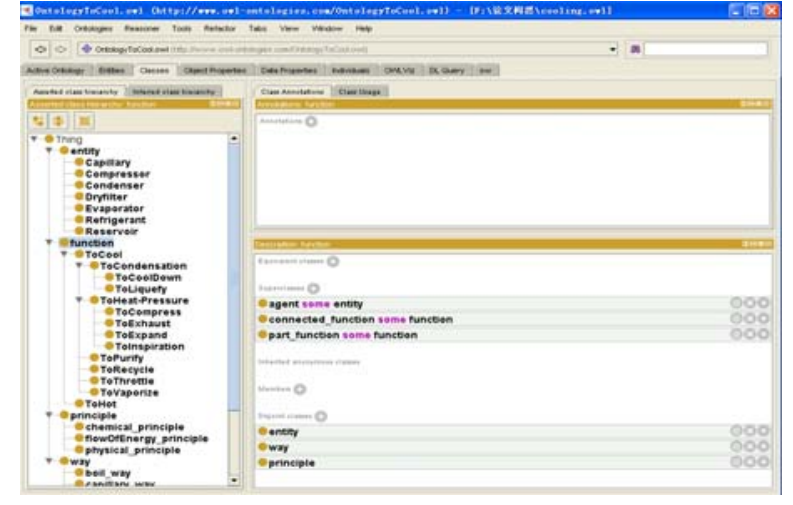

Figure 3. Ontology built with protégé

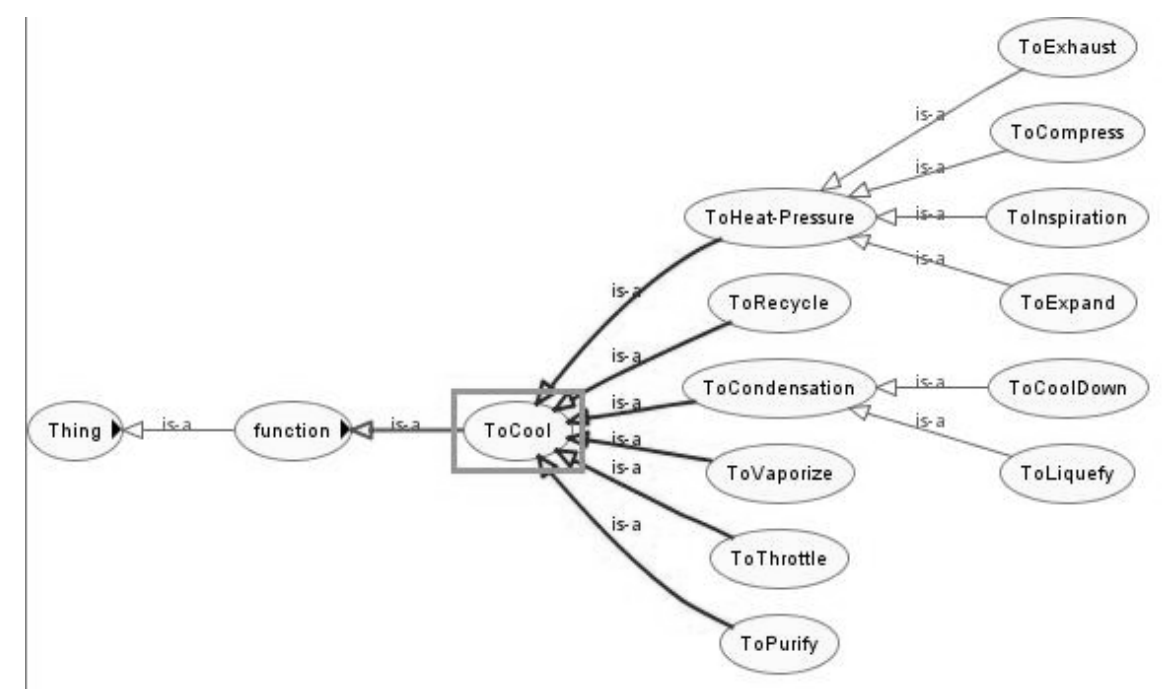

Figure 4. Part of function graph generated by protégé 


\section{Implementation of query process}

The functions of component index designed in this paper have been implemented as following:

First, based on required function input by user, the entity with required function (e.g. equipment) can be retrieved and the tree graph is given as well;

Second, user input name of component, all functions of this component is retrieved, and some relevant information is also given.

This project is developed in Java language, call Jena package designed in the HP laboratory to achieve query function, and the SPARQL (Simple Protocol and RDF Query Language) ontology query language technology has been used. SPARQL is a query language and a protocol of obtaining data for RDF, it is developed for RDF from W3C to establish data model. But can be used for any information resources expressed using RDF. Currently SparQL protocol and RDF query language (SparQL) is the working draft or recommended standards of W3C, they are still under discussion. SparQL query language is constructed on the previous RDF (such as rdfDB, RDQL and SeRQL) and has some valuable new features.

A rich vocabulary is provided to establish a specific product function decomposition tree by searching process. Users can selectively draw a specific product function decomposition tree based on the contents retrieved through searching process, at the same time; a optimal functional decomposition tree can be obtained through comparing with the previous similar products. This function provides the basis for later development solution tree software platform. Below is a partial result shows the query, but the whole software platform need to be improved.

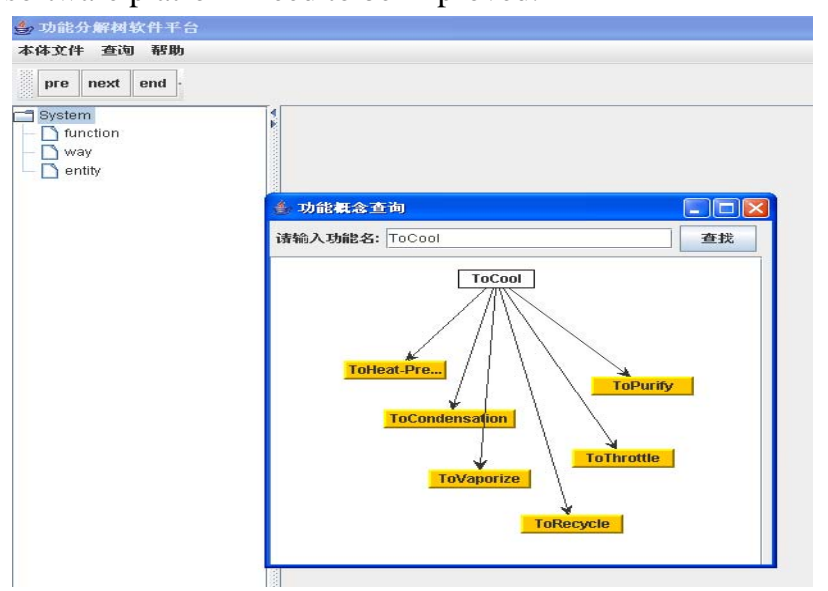

Figure 5. A screenshot of function decomposition tree software platform

\section{SUMMARY}

Based on analysis of problems of design knowledge sharing in the current manufacturing industry and research of function knowledge ontology, the function retrieval based on knowledge ontology has been implemented, provides the basis for further drawing functional decomposition tree as well. Basically the problem of design knowledge sharing in current manufacturing industry can be solved through functional decomposition tree software platform. Meanwhile, it is found that research of function knowledge retrieval based on ontology has much of the subsequent work, such as ontology further improvement, the function reasoning, functional decomposition tree software platform establishment etc.

\section{REFERENCES}

[1] Li Jian etc., Product design process based on Function Research, Computer integrated manufacturing system -CIMS , 2002.

[2] Shi Dongyan , Study of Conceptual Design Solution Process Based on Functio, Harbin Institute of Technology, 2006.

[3] Wang Youyuan, Product information modeling of conceptual design based on Ontology, Journal of Wuhan University (Engineering and Technology Edition), 2006.

[4] Yoshinobu Kitamur, Naoya Washio,Yusuke Koji. An Ontology-Based Annotation Framework for Representing the Functionality of Engineering Devices.

[5] Yoshinobu Kitamura,Toshinobu Sano,Kouji Namba,and Riichiro Mizoguchi. A Functional Concept Ontology and Its Application to Automatic Identification of Functional Structures.

[6] Munehiko Sasajima, Yoshinobu Kitamura, Mrrsuru Ikeda and Riichiro Mizoguchi. A Representation Language for Behavior and Function: FBRL.

[7] Yan Wei etc. Design and implementation of component functional retrieval based on Ontology,

Computer Technology and Development, 2009.

[8] Yoshinobu Kitamura,Yusuke Koji and Riichiro Mizoguchi, An Ontological Model of Device Function and Its Deployment for Engineering Knowledge Sharing.

[9] Yoshinobu Kitamura and Riichiro Mizoguchi,Ontology-based Functional-Knowledge Modeling Methodology and its Deployment.

[10] Yoshinobu Kitamura and Riichiro Mizoguchi,Ontology-based Description of Functional DesignKnowledge and its Use in a Functional Way Server. 